\title{
New Lewis Structures through the application of the Hypertorus Electron Model
}

\author{
Omar Yépez \\ yepezoj@gmail.com
}

November 14, 2010

\begin{abstract}
The hypertorus electron model is applied to the chemical bond. As a consequence, the bond topology can be determined. A linear correlation is found between the normalized bond area and the bond energy. The normalization number is a whole number. This number is interpreted as the Lewis's electron pair. A new electron distribution in the molecule follows. This discovery prompts to review the chemical bond, as it is understood in chemistry and physics.
\end{abstract}

\section{Introduction}

The topological analysis of the electron density has provided useful information about the bonding in a molecule. However, not much progress has been made to reveal the fundamental features of chemical bonding postulated by Lewis, i.e. the electron pair. According to Lewis structures there are bonding electron pairs in the valence shell of an atom in a molecule, and there are also nonbonding pairs or lone pairs in the valence shell of many of the atoms in a molecule. So far, it has not been seen any evidence of electron pairing in the topological analysis of the electron density. An increased concentration of electron density is observed between the two bonded atoms. Which could be interpreted as the electron density equivalent of a Lewis bonding pair [1]. Nevertheless, hitherto there is no way to be sure about it. The same occurs about the existence of lone pairs. This same book arrives to the conclusion that electron pairs are not always present in molecules, and even when they are, they are not as localized as the approximate models may suggest [2].

Therefore, a method to measure the number of electrons that participate in the bond will definetely probe or not the existance of Lewis's electron pairs. 
On 1996 the shape of the deuteron were reported. The deuteron presents three different shapes: a torus, an sphere inside another sphere and two separated spheres [3]. These are the three possible intersections between a three dimensional space, that cut a hypertorus in half $[4,5,6]$. Of course, this definition is not reported in reference [3]. However, this is very important because these are the same shapes observed in every single molecule's laplacian of the electron density. Lack of identifying these shapes with the electron misleads the molecule's topological analysis.

This paper uses this new shapes in the topological analysis of different diatomic molecules and $\mathrm{CO}_{2}$. Thanks to this, the topology of the chemical bond is properly identified. The area of this bond topology is correlated with its bond energy. Only when the bond area is divided by a whole number, a linear correlation between the bond area and the bond energy occurs. This whole number is interpreted as the electron pair. Consequently, an electron distribution in the molecule is possible. First time evidences of the Lewis electron's pair are found.

\subsection{Electron Pair Topology}

Valence electrons or lone pairs will be observed in the bond using the structures observed in Fig. 1, namely: the two separated spheres $(t s s)$, the torus $(t)$ and the sphere in a sphere $(s s)$.

Valence electrons participating in the $\sigma$ bond occur in the two separated sphere structure, $t s s$. This structure will involve the atoms compromised in this chemical bond. Double, triple and quadruple bonds also adopt this structure. As the electronegativity of the nucleus increases, the valence electrons tend to form a toroidal structure around the atom. These electrons acts like lone pairs and they do not participate in $\sigma$ bonds. If the atom electronegativity is higher the lone pair can adopt a ss structure.

\section{Experimental}

By cutting the silhouette of the two separated sphere structure, involving the bonded atoms. The bond areas were determined from the contour map of the Laplacian of its charge density. An example of such silhouette (green lines) can be observed in Fig. 4 for the fluorine molecule. They were printed on paper and weighted. The bond length was used to calibrate the area measured in each bond. Then, these areas were correlated with their respective bond energies.

The contour map of the Laplacian of the charge density for fluorine, $\mathrm{F}_{2}$ and dicarbon, $\mathrm{C}_{2}$ molecules were found in ref. [7]. Oxygen, $\mathrm{O}_{2}$ was found coordinated to a molibdenum atom in ref. [8] and free in ref. [9]; in the latter reference diboron, $\mathrm{B}_{2}$ was also found. Nitrogen, $\mathrm{N}_{2}$ is from [10]. Carbon monoxide, $\mathrm{CO}$ was found in ref.[11]. Nitrogen monoxide, NO 


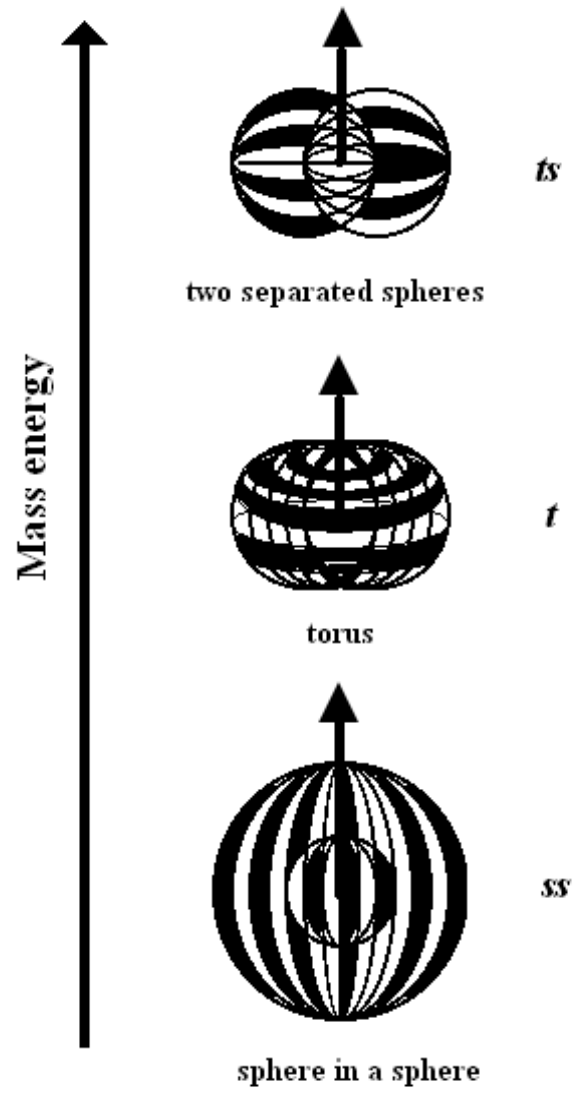

chch

Figure 1: Observables structures of the hypertorus electron. The magnetic moment in each structure begins in the geometric center of the structure, see ref.[4]. 


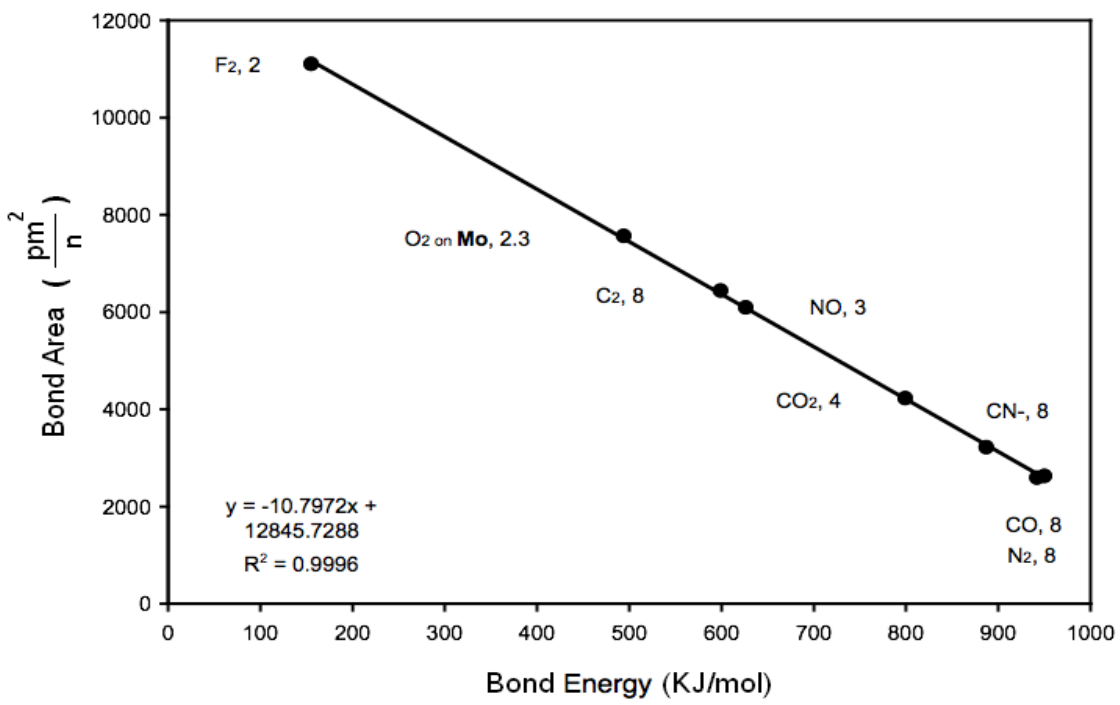

chch

Figure 2: Correlation between bond area and bond energy for different diatomic molecules and $\mathrm{CO}_{2}$.

in ref.[12]. Cyanide $\mathrm{CN}^{-}$was found in ref.[13] and carbon dioxide $\mathrm{CO}_{2}$ was found in ref. [14].

\section{Results}

Fig. 2 shows a straightforward correlation between the bond area divided by a number $\mathbf{n}$ and the bond energy of each bond. This number $\mathbf{n}$ is a whole number and it is interpreted as the number of electrons involved in the bond. It has to be stressed that the y axis location for each experimental point is very sensitive to the number $\mathbf{n}$. Fractions of this number makes the $\mathrm{r}^{2}$ get lower than 0.999. It is clear that as the normalized bond area diminished, the bond energy increases.

\subsection{Homonuclear diatomic molecules}

Fig. 3 shows the fluorine molecule. The sphere in a sphere structure is clearly observed at the center of each $\mathrm{F}$ atom. It is due to the helium core. The next six electrons are in the toroidal structure around such helium core. As observed in Fig. 2, the F-F bond has two electrons. The two bonding electrons belong to both nuclei in a tss structure. Due to 


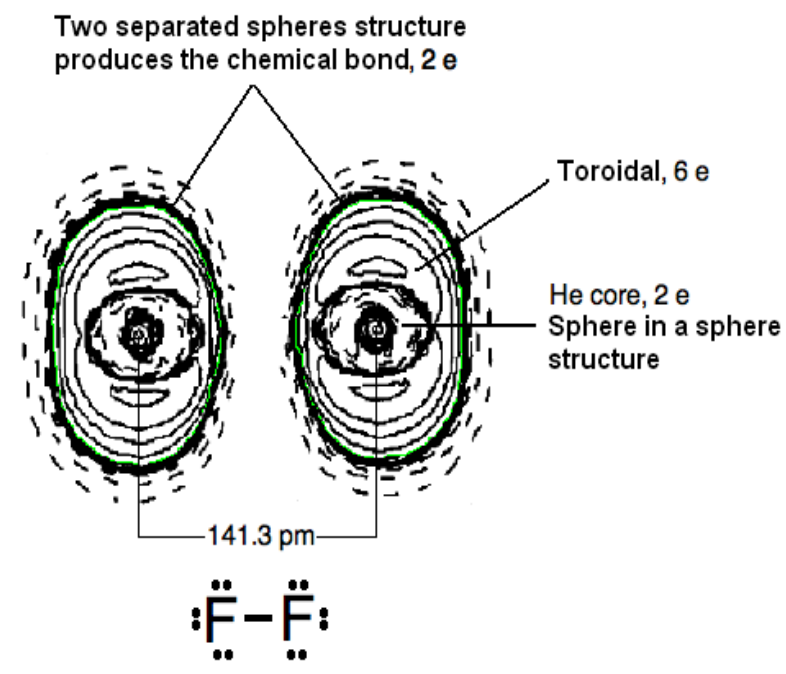

chch

Figure 3: a) fluorine molecule. There is no discernible structures between the atoms. The different electron's structures are indicated. The green line shows how the bond was cut.

this bonding, there is no discernible structure between the F atoms. Therefore, one can still put an stroke between these two atoms, understanding that there is a bond through this structure. Hence F-F is all right. The dots around each F atom just denotes the pairing of each atom 6 toroidal electrons. This is the usual Lewis structure.

Oxygen, $\mathrm{O}_{2}$. Fig. 4 shows that the oxygen molecule highly resembles the fluorine one. The $\mathbf{n}$ number was not a whole number giving 2.3. The uncoupled electrons in each oxygen atom will produce a magnetic attraction in the line of the bonding. Probably, this may distort the molecule in a way to make it digresses from the experimental trend observed. However, the resemblance to the fluorine molecule and the closeness of the $\mathbf{n}$ number to 2 , strongly suggests that the number of electrons involve in the $\mathrm{O}-\mathrm{O}$ bond is 2 .

As a consequence, the toroidal structure on the oxygen's helium core, previously observed in $\mathrm{F}_{2}$, necessarily have 5 electrons each. This odd number means two uncoupled magnetic momenta. One in each Oxygen atom. They will align as indicated in the figure. This will create a net magnetic moment in the molecule, i.e. oxygen molecule is paramagnetic.

The magnetic attraction is rendering a shorter area in this molecule. Probably, this is why this molecule is away from the general trend observed in Fig. 2. Dividing between a larger $\mathbf{n}$ number is just compensating this magnetic disturbance. In other words, to have an $\mathbf{n}=2$ in this molecule, the energy of the $\mathrm{O}-\mathrm{O}$ bond should be $410 \mathrm{~kJ} / \mathrm{mol}$ and not the experimental $494 \mathrm{KJ} / \mathrm{mol}$.

This magnetic disturbance is larger in $\mathrm{B}_{2}$ (not shown). This molecule is also paramagnetic, thus it should have two uncoupled electrons on each boron atom, just as in oxygen molecule. Therefore, the $\mathbf{n}$ for this molecule should be four. Contrarily, 5.5 was found for this molecule 


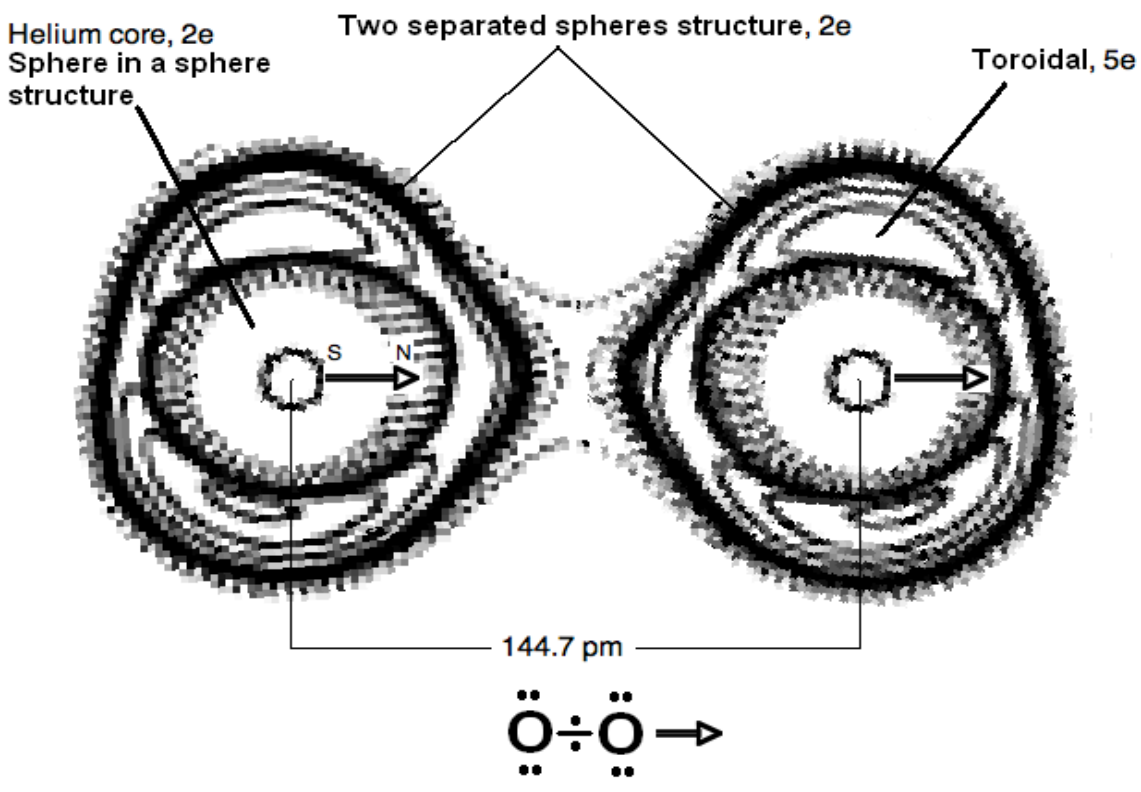

chch

Figure 4: Oxygen molecule coordinated by a Mo atom in ref. [8] . The different electron's structures are indicated. The magnetic moments are shown with the arrow with North and South poles.

to match the trend observed in Fig. 2. This denotes a stronger magnetic disturbance in the bond.

There have not been any consensus about how the oxygen's Lewis structure should be written. The molecule's paramagnetism does not help. This is because an uncouple electron structure has to be written, somehow contradicting Lewis pairing hypothesis. $\mathrm{O}-\mathrm{O}, \mathrm{O}=\mathrm{O}$ and $\mathrm{O} \div \mathrm{O}$ has been proposed. From these structures, the more pertinent is $\mathrm{O} \div \mathrm{O}$ because the dots are the two uncouple electrons observed in figure 4. the Lewis structure observed in figure 4 is equivalent. The only difference is the arrow that describe a net magnetic moment and the location of each magnetic momentum.

Nitrogen, $\mathbf{N}_{2}$. As it is noticeable from Fig. 5, the nitrogen atoms are not separated. This is probably due to the lower electronegativity in comparison with fluorine and oxygen molecules. The well defined tss structure previously observed for fluorine and oxygen disappears. Giving way to the same structure but with its spheres more collapsed; this is covering both nitrogen atoms.

According to the results from Fig. 2, four of the five nitrogen valence electrons are compromised in the N-N bond. Since this molecule is diamagnetic, it is believed that the two remaining electrons overlaps forming a toroidal lone pair structure around the N-N bond. This ring will occur in the midpoint between the bonding nitrogen. Structures like this has been observed, for example in the acetylene molecule [15]. As a consequence of this electron 


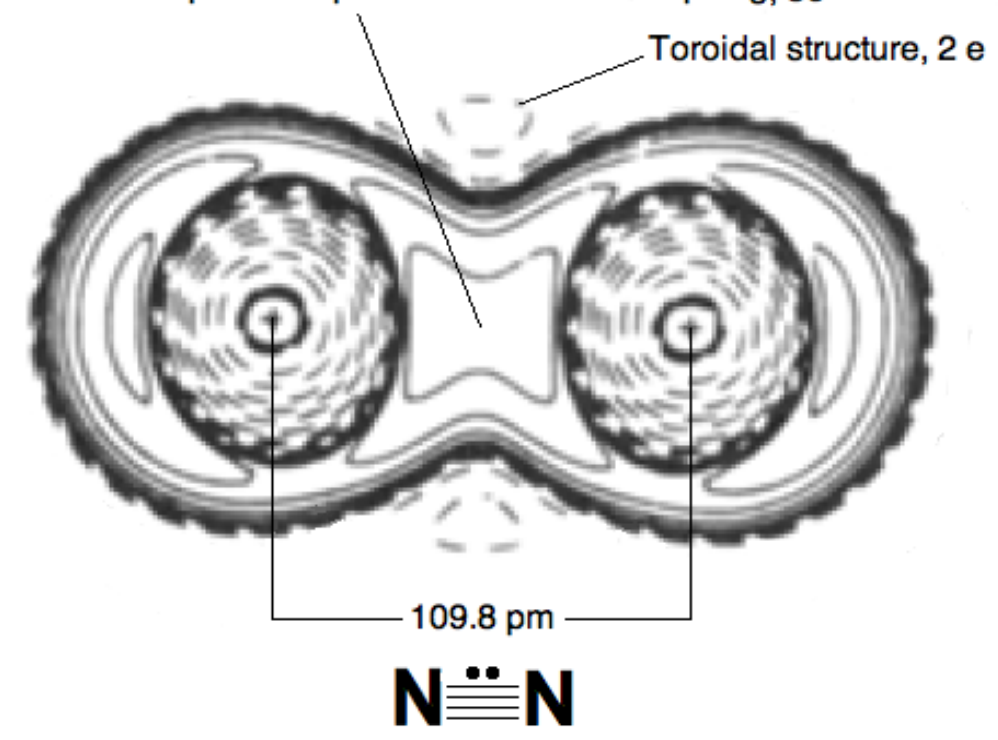

chch

Figure 5: Nitrogen molecule.

distribution, all nitrogen's five valence electrons are overlaped and this is why this molecule presents the highest bond energy in the series F, O, N, C.

The usual Lewis structure is a triple bond between the nitrogens and two lone pairs, one at each nitrogen atom. However, this molecule has one of the highest bond energies and also the smaller bond area measured from the pool of molecules tested. Therefore, it should not surprise that a very high number of valence electron overlap for this bond. Furthermore, there is no structures in Fig. 5 to justify the presence of lone pairs on neither $\mathrm{N}$ atom. As it was observed in $\mathrm{F}-\mathrm{F}$ or in $\mathrm{O} \div \mathrm{O}$. Hence, the Lewis structure picture in Fig. 5 with four strokes and the lone pair at the middle of the N-N bond is a new Lewis structure.

Dicarbon, $\mathbf{C}_{2}$. Fig. 6 presents an even less collapsed tss structure in comparison with $\mathrm{N}_{2}$. This is due to the available valence electrons to bond and to the lower electronegativity that carbon has. The $\mathrm{C}-\mathrm{C}$ bond in dicarbon involves all valence electrons from each carbon, i.e. 8. The diamagnetism of this molecule revels that all its bond electrons are magnetically coupled.

Again, no lone pair structures are noticeable in this molecule. Hence, the Lewis structure depicted in Fig. 6 is new.

Upon comparing these four molecules, one can arrive to the conclusion that the chemical bond is mostly performed by this tss structure. As the electronegativity of the bonded atoms diminishes, more electrons are involved in the bond. 


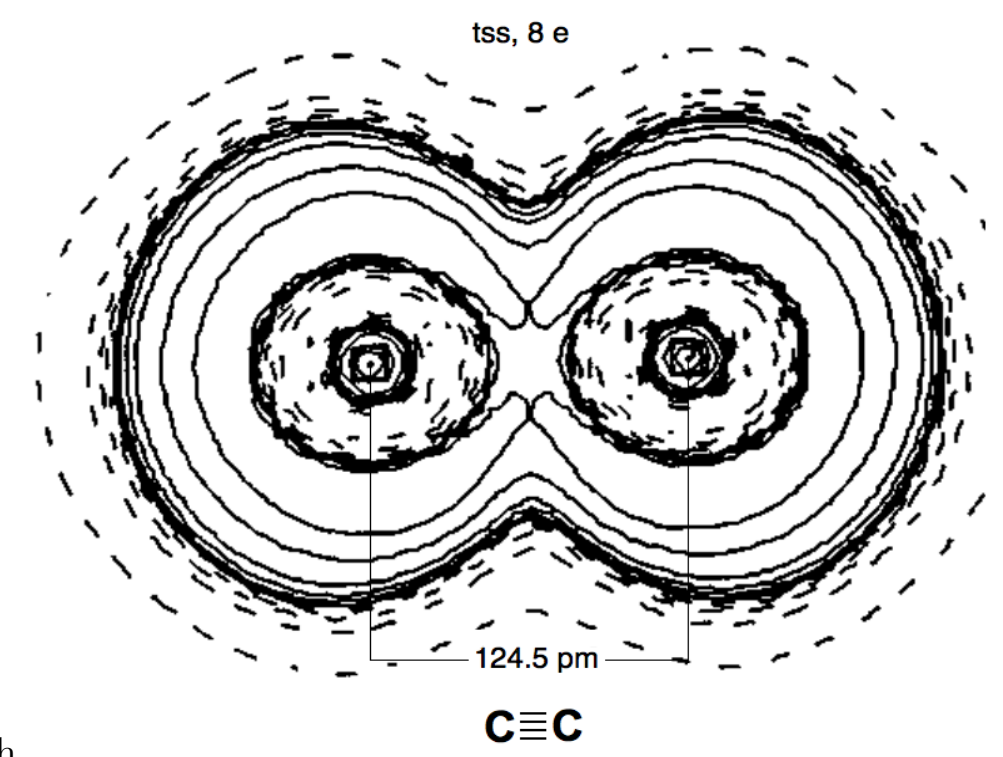

Figure 6: Dicarbon molecule

\subsection{Heteronuclear molecules}

Carbon monoxide, CO. As observed in Fig. 2, C-O bond involves 8 electrons. Accordingly, Fig. 7 presents the electron distribution in CO. From the 10 valence electrons to share: 4 from the carbon and 4 from the oxygen overlap in the $\mathrm{C}-\mathrm{O}$ bond. The other 2 oxygen's valence electrons are in a lone pair. This is the ss structure over the oxygen.

This molecule is isoelectronic with $\mathrm{N}_{2}$. However, the difference between the atoms' electronegativity makes the lone pair to form over the oxygen. In the case of $\mathrm{N}_{2}$, there is no difference in electronegativity, thus the toroidal lone pair has to be at the middle of the N-N bond.

The current Lewis structure of CO is a triple bond between the carbon and the oxygen and one lone pair on each atom. Somehow trying to achieve the octet rule. The new Lewis structure is a quadruple bond for the $\mathrm{C}-\mathrm{O}$ bond and one lone pair only on the oxygen (see Fig.7).

Finally, there is a controversy about the dissociation energy of CO. The values can be 881, 926, 949, 941 or $1070 \mathrm{KJ} / \mathrm{mol}$ coming from different kind of experiments [16]. In the case of Fig. 2, the value 926 from electron impact experiments or $949 \mathrm{KJ} / \mathrm{mol}$ from predissociation data produced the best linear correlation with the other molecules of the group.

Cyanide, $\mathbf{C N}^{-}$. As in the case of carbon monoxide, the $\mathrm{C}-\mathrm{N}$ bond involves 8 electrons. Fig. 8 presents the electron distribution in the molecule: 4 valence electrons from carbon and 4 more from the nitrogen make this bond in a tss structure. The nitrogen however, remains with one uncouple electron. Since this molecule is diamagnetic, an extra electron is needed to coupled and cyanide finished with a negative charge. This charge is in the lone 


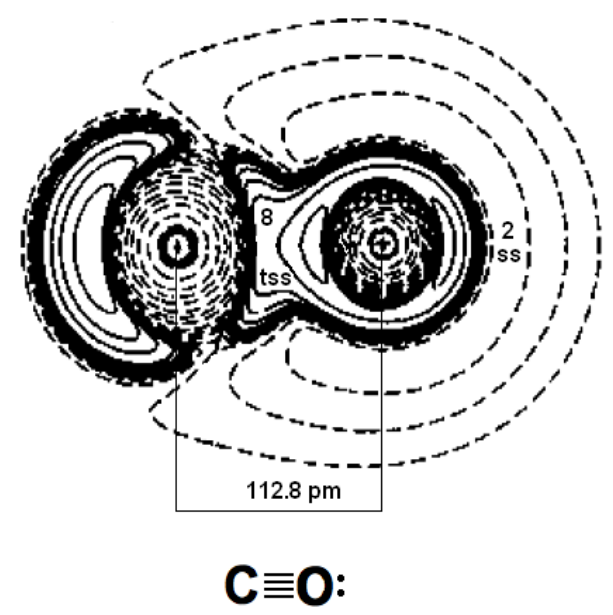

Figure 7: Carbon monoxide.

pair, clearly observed as an ss structure on the nitrogen. This occurs on the nitrogen atom because it is more electronegative than carbon. This molecule is also isoelectronic with CO and $\mathrm{N}_{2}$. Again, the current Lewis structure is a triple bond between the carbon and the nitrogen and two lone pairs; one on each atom. This is to try to achieve the octet rule. Again, just like in the CO molecule, the new Lewis structure is a quadruple bond and the lone pair repeats on the more electronegative atom, the nitrogen.

Nitrogen monoxide, NO. Fig. 9 presents NO molecule. As observed in Fig. 2, the N-O bond involve three electrons. This will imply that one of those three electrons is not magnetically coupled with the other two and therefore, this molecule will be paramagnetic. In this overlap of three electrons, the nitrogen shares 1 and the oxygen shares 2 . By this way, the nitrogen can couple the other 4 electrons as toroidal lone pair. This is indicated in the figure. The oxygen will arrange its other 4 electrons in the same manner. The current Lewis structure places an isolated uncoupled electron on the nitrogen. The current Lewis structure depicts an uncoupled electron on the nitrogen and a double bond between the nitrogen and the oxygen. The new Lewis structure left the odd electron in the N-O bond. Thus, this would be an example of a three electron bond and this bond is paramagnetic. Thus, the new Lewis structure draws a magnetic arrow over the single N-O bond. The two lone pair on each atom are also depicted.

Carbon dioxide $\mathbf{C O}_{2}$. Fig. 10 shows that the 4 valence electrons of carbon are used at 


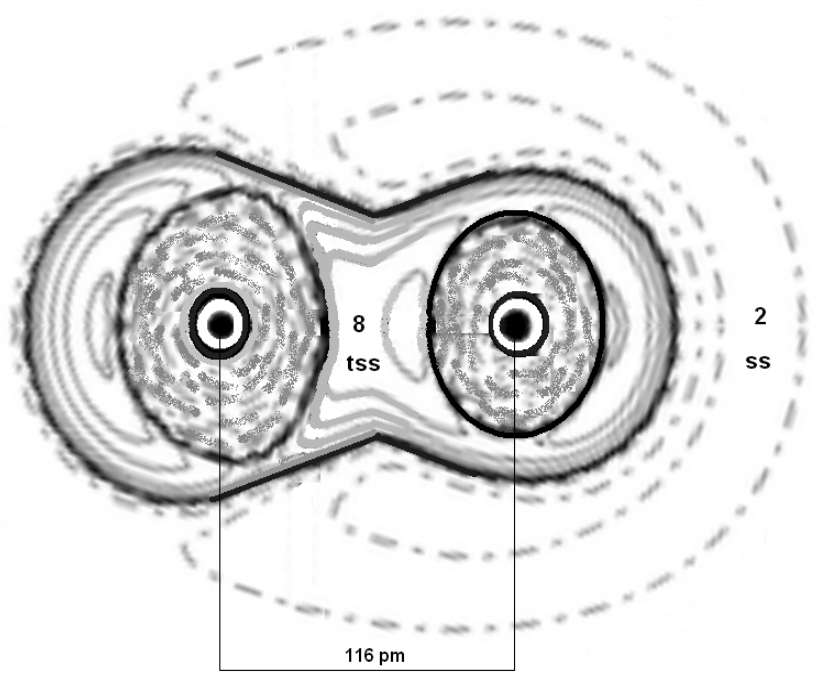

\section{CDN:}

chch

Figure 8: Cyanide molecule.
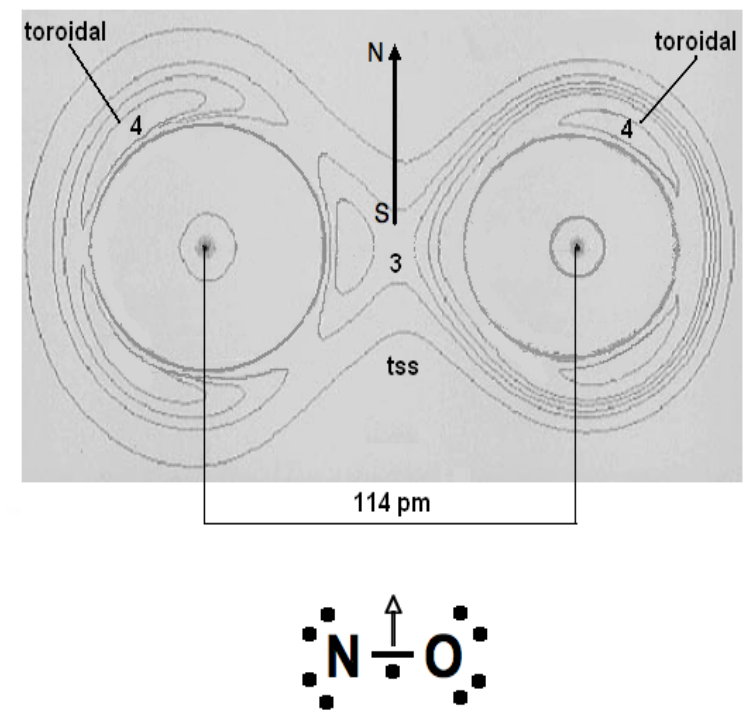

chch

Figure 9: Nitrogen monoxide molecule. 


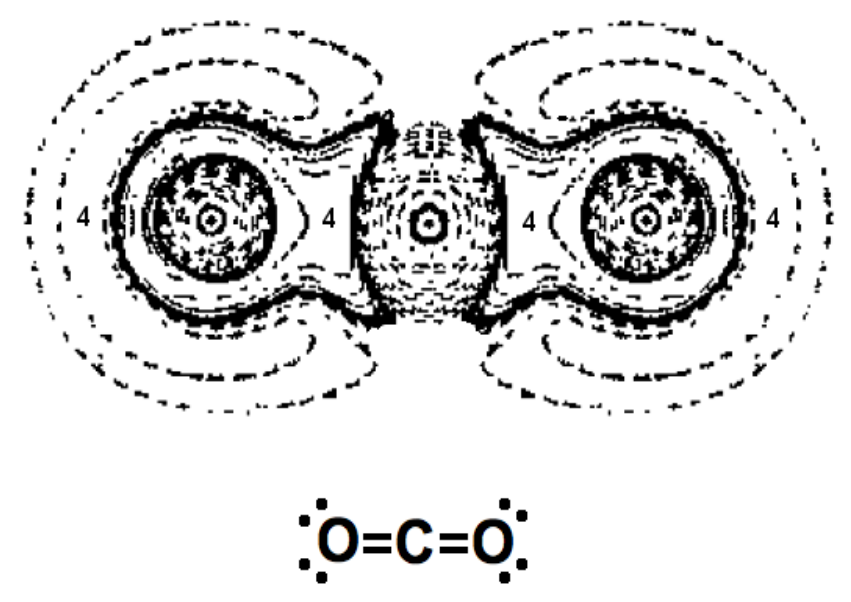

chch

Figure 10: Carbon dioxide. $\mathrm{CO}_{2}$.

each side of the molecule to produce two C-O bonds with 4 electrons each. The remaining 4 electrons of the oxygen go to a lone pair over each oxygen atom. This molecule fits the octet rule and does not suffer any modification in its Lewis structure.

\section{Final Remarks}

The topological evidence points that Lewis hypothesis is right. Electrons do bond in pairs, lone pairs do form on the most electronegative atom. However, the octet rule applied to fewer cases $\mathrm{F}_{2}$, to $\mathrm{N}_{2}$ (if the share lone pair is not considered as part of the bond) and in $\mathrm{CO}_{2}$. The rest of the molecules does not obey such a rule. Instead, $\mathrm{N}_{2}, \mathrm{C}_{2}, \mathrm{CO}$ and $\mathrm{CN}^{-}$ form a quadruple bond. When there is a lone pair, it locates according to the net molecule's electronegativity. This probably means that the octet rule has to go, i.e., atoms not necessarily share valence electrons in order to achieved a noble gas structure. 


\section{References}

[1] R. J. Guillespie and P.L.A. Popelier, "Chemical Bonding and Molecular Geometry, from Lewis to electron densities", Oxford University Press, New York, 2001, Chapter 7.

[2] R. J. Guillespie and P.L.A. Popelier, "Chemical Bonding and Molecular Geometry, from Lewis to electron densities", Oxford University Press, New York, 2001, p.179.

[3] J.L. Forest, V. R. Pandharipande, Steven C. Pieper, R. B. Wiringa, R. Schiavilla and A. Arriaga, Phys. Rev. C, 54(1996)646667.

[4] O. Yépez, arXiv:physics/0401153 v1 28 Jan 2004.

[5] O. Yépez, arXiv:physics/ 0510168 v1 18 Oct 2005.

[6] O. Yépez, arXiv:physics/0701346 v1 31 Jan 2007.

[7] Wai-To Chan and I. P. Hamilton, J. Phys. Chem. 108 (1998) 2473.

[8] P. Macchi, A. J. Schultz, F.K. Larsen, and B. B. Iversen, J. Chem. Phys. 105 (2001) 9231.

[9] R. F.W. Bader, "Atoms in Molecules, a Quantum Theory", Clarendon Press, Oxford, 1990, pp. 294.

[10] R. F.W. Bader, Chem. Rev., 91 (1991) 893.

[11] R. F.W. Bader, S. Johnson, T. H. Tang and P.L.A. Popelier, J. Phys. Chem., 100 (1996) 15398.

[12] Aray Y., J. Rodriguez and R. Lopez-Boada, J. Phys. Chem. A, 101 (1997) 2178.

[13] M. C. Daza, J. A. Dobado, J. Molina and J. L. Villaveces, Phys. Chem. Chem. Phys., $2(2000) 4089$.

[14] R.F.W. Bader and T.A. Keith, J. Chem. Phys. 99 (1993) 3683.

[15] S. R. Gadre, P. K. Bhadane, Resonance 4(1999)14.

[16] Pauling L. and Sheehan W. F., Proc. N. A. S., 35 (1949) 359. 